\title{
Third-Order Generalized Discontinuous Impulsive Problems on the Half-Line
}

\section{Feliz Minhós ${ }^{a, b}$ and Rui Carapinha ${ }^{b}$}

${ }^{a}$ Departamento de Matemática, Escola de Ciências e Tecnologia, Universidade de Évora, Rua Romão Ramalho 59, 7000-671 Évora, Portugal

${ }^{b}$ Centro de Investigação em Matemática e Aplicações (CIMA), Instituto de Investigação e Formação Avançada, Universidade de Évora, Rua Romão Ramalho 59, 7000-671 Évora, Portugal

E-mail(corresp.): fminhos@uevora.pt

E-mail: gene.destro@gmail.com

Received April 23, 2020; revised August 31, 2021; accepted September 1, 2021

Abstract. In this paper, we improve the existing results in the literature by presenting weaker sufficient conditions for the solvability of a third-order impulsive problem on the half-line, having generalized impulse effects. More precisely, our nonlinearities do not need to be positive nor sublinear and the monotone assumptions are local ones. Our method makes use of some truncation and perturbed techniques and on the equiconvergence at infinity and the impulsive points. The last section contains an application to a boundary layer flow problem over a stretching sheet with and without heat transfer.

Keywords: impulsive problems, upper and lower solutions, equiconvergence, boundary layer flow.

AMS Subject Classification: 34B37; 34B40; 34B15.

\section{Introduction}

In this work we deal with a boundary value problem composed of the thirdorder differential equation on the half real line

$$
u^{\prime \prime \prime}(t)=f\left(t, u(t), u^{\prime}(t), u^{\prime \prime}(t)\right), t \in[0,+\infty),
$$

where $f:[0,+\infty) \times \mathbb{R}^{3} \rightarrow \mathbb{R}$ is an $L^{1}-$ Carathéodory function, together with the boundary conditions

$$
u(0)=A, u^{\prime}(0)=B, u^{\prime \prime}(+\infty)=C,
$$

Copyright (C) 2021 The Author(s). Published by Vilnius Gediminas Technical University This is an Open Access article distributed under the terms of the Creative Commons Attribution License (http://creativecommons.org/licenses/by/4.0/), which permits unrestricted use, distribution, and reproduction in any medium, provided the original author and source are credited. 
with $A, B, C \in \mathbb{R}, u^{\prime \prime}(+\infty):=\lim _{t \rightarrow+\infty} u^{\prime \prime}(t)$ and the impulsive effects given by the generalized functions

$$
\begin{aligned}
\Delta u\left(t_{k}\right) & =I_{0 k}\left(t_{k}, u\left(t_{k}\right), u^{\prime}\left(t_{k}\right), u^{\prime \prime}\left(t_{k}\right)\right), \\
\Delta u^{\prime}\left(t_{k}\right) & =I_{1 k}\left(t_{k}, u\left(t_{k}\right), u^{\prime}\left(t_{k}\right), u^{\prime \prime}\left(t_{k}\right)\right), \\
\Delta u^{\prime \prime}\left(t_{k}\right) & =I_{2 k}\left(t_{k}, u\left(t_{k}\right), u^{\prime}\left(t_{k}\right), u^{\prime \prime}\left(t_{k}\right)\right),
\end{aligned}
$$

with $0=t_{0}<t_{1}<t_{2}<\ldots<t_{k}<\ldots, k \in \mathbb{N}$, such that $\lim _{k \rightarrow+\infty} t_{k}=+\infty$ and $I_{i k}:[0,+\infty) \times \mathbb{R}^{3} \rightarrow \mathbb{R}$ Catathéodory sequences for $i=0,1,2$ and $k \in \mathbb{N}$.

We point out that the technique presented in this paper can be easily adapted, with obvious changes, to $n^{\text {th }}$ order problems of the type

$$
\begin{aligned}
u^{(n)}(t) & =f\left(t, u(t), \ldots, u^{(n-1)}(t)\right), t \in[0,+\infty), \\
u^{(i)}(0) & =A_{i}, \quad u^{(n-1)}(+\infty)=B, A_{i}, B \in \mathbb{R}, i=0,1, \ldots, n-2, \\
\Delta u^{(j)}\left(t_{k}\right) & =I_{i k}\left(t_{k}, u\left(t_{k}\right), \ldots, u^{(n-1)}\left(t_{k}\right)\right), j=0,1, \ldots, n-1, k \in \mathbb{N} .
\end{aligned}
$$

The option for order three here, is due to clearance reasons, to highlight the method and not make the reading more difficult with heavy notation.

These higher-order boundary value problems with asymptotic conditions can model some real phenomena as gas pressure in a semi-infinite porous medium, draining or coating fluid-flow problems, and other evolution of physical processes. Likewise, they are useful in more theoretical studies such as on nonlinear elliptic equations, to prove the existence of radially symmetric solutions, or heteroclinic and homoclinic solutions of differential equations, or coupled systems of differential and integral equations. As related works we mention, for instance, [2,5,16,17, 18, 19, 26, 27].

As the infinite interval is noncompact, the discussion about sufficient conditions for the solvability of boundary value problems on the half-line is more delicate. In the literature the main methods to obtain existence results are the extension of continuous solutions on finite intervals via a diagonalization process, lower and upper solutions and fixed point theorems in Banach weighted spaces (see $[3,4,11,30]$ and their references).

Impulsive problems, that is, situations where sudden variations happens, have had an important development in last decades, mostly due to their applicability to real life phenomena. See, for example, [1, 12, 13, 14, 16, 21, 24, 25, 28] and the references therein.

In [29], the authors consider a problem similar to (1.4)-(1.5) where the nonlinearity and all the impulsive functions must be sublinear and nondecreasing in all space variables. The existence of positive solutions is proved using cone theory and Mönch's fixed point theorem, together with a monotone iterative technique.

Motivated by this work, we study problem (1.1)-(1.3) under weaker conditions, not only on the nonlinearity but also on the impulsive functions. Indeed, being more specific:

- the nonlinearity $f$ is an $L^{1}$ - Carathéodory function, meaning that it could be discontinuous in time and, eventually, superlinear near the origin 
or at $+\infty$. Moreover, there is not a monotone assumption on $f$ in the highest order derivative, while in the other variables there is only the restriction of a local monotony in some strip;

- the impulsive functions $I_{i k}$, with $i=0,1,2$, are locally monotone, that is, the monotonicity is required only on a strip. Moreover, the sequence $I_{2 k}$ has a different monotone behavior of [29] and there is no monotone assumption at all on the highest order variable;

- the solutions may have negative values.

Our method relies on the lower and upper solutions technique, which reveals to be adequate to these impulsive boundary value problems, adding to the existence of solution its localization and some qualitative data on its behavior as well. We apply some truncation and perturbation techniques suggested, for example, in $[6,9,10]$, together with equiconvergence at $+\infty$ and on the impulsive points, as it appears in [8].

The paper is organized in the following way: Section 2 contains the definition of the Banach spaces, the corresponding weighted norms, and other auxiliary results as well. In Section 3 we present the main theorem: an existence and localization result, where we prove the existence of at least one solution, and some bounds on the first and second derivatives. The last section, has an application to a boundary layer flow problem over a stretching sheet with and without heat transfer.

\section{Definitions and auxiliary results}

A key argument of our method is based on a weighted space with some asymptotic assumptions.

Consider the spaces

$$
P C^{2}[0,+\infty]=\left\{\begin{array}{c}
u: u \in C^{2}([0,+\infty] ; \mathbb{R}) \text { for } t \neq t_{k}, u^{(i)}\left(t_{k}\right)=u^{(i)}\left(t_{k}^{-}\right), \\
u^{(i)}\left(t_{k}^{+}\right) \text {exists for } k=1,2, \ldots, m, \text { and } i=0,1,2
\end{array}\right\}
$$

and

$$
X=\left\{x \in P C^{2}[0,+\infty): \lim _{t \rightarrow+\infty} \frac{x^{(i)}(t)}{w_{i}(t)} \text { exists, } i=0,1,2\right\}
$$

with $w_{i}(t)=1+t^{2-i}$ and the norm $\|y\|=\max \left\{\|y\|_{0},\|y\|_{1},\|y\|_{2}\right\}$, where

$$
\|y\|_{0}=\sup _{0 \leq t<+\infty}\left\{\frac{|y(t)|}{1+t^{2}}\right\},\|y\|_{1}=\sup _{0 \leq t<+\infty}\left\{\frac{\left|y^{\prime}(t)\right|}{1+t}\right\},\|y\|_{2}=\sup _{0 \leq t<+\infty}\left\{\frac{\left|y^{\prime \prime}(t)\right|}{2}\right\} .
$$

Therefore, $(X,\|\|$.$) is a Banach space.$

The nonlinearities will have the regularity of $L^{1}-$ Carathéodory functions defined as it follows:

Definition 1. A function $f:[0,+\infty) \times \mathbb{R}^{3} \rightarrow \mathbb{R}$ is $L^{1}-$ Carathéodory if it satisfies 
i) for each $(x, y, z) \in \mathbb{R}^{3}, t \mapsto f(t, x, y, z)$ is measurable on $[0,+\infty)$;

ii) for almost every $t \in[0,+\infty),(x, y, z) \mapsto f(t, x, y, z)$ is continuous in $\mathbb{R}^{3}$;

iii) for each $\rho>0$, there exists a positive function $\psi_{\rho} \in L^{1}[0,+\infty)$ such that, for $\max \left\{\|x\|_{0},\|y\|_{1},\|z\|_{2}\right\}<\rho$,

$$
|f(t, x, y, z)| \leq \psi_{\rho}(t), \text { a.e. } t \in[0,+\infty) .
$$

The impulsive effects are given in terms of sequences of functions as in next definition.

Definition 2. A sequence $\left(w_{n}\right)_{n \in \mathbb{N}}$ is a Carathéodory sequence if

(i) for each $(x, y, z) \in \mathbb{R}^{3},(x, y, z) \rightarrow w_{n}(x, y, z)$ is continuous for all $n \in \mathbb{N}$;

(ii) for each $\rho>0$, there are nonnegative constants $\lambda_{n \rho} \geq 0$ with $\sum_{n=1}^{+\infty} \lambda_{n \rho}<$ $+\infty$ such that for $|x|<\rho\left(1+t^{2}\right),|y|<\rho(1+t),|z|<2 \rho$, for $t \in[0,+\infty)$, we have

$$
\left|w_{n}(x, y, z)\right| \leq \lambda_{n \rho} \text {, for every } n \in \mathbb{N} \text {. }
$$

Next lemma gives the exact solution for the associated linear and homogeneous problem:

Lemma 1. If $e \in L^{1}[0,+\infty)$, then the boundary value problem

$$
\left\{\begin{array}{l}
u^{\prime \prime \prime}(t)=e(t), \quad t \in(0,+\infty), \\
u(0)=A, u^{\prime}(0)=B, u^{\prime \prime}(+\infty)=C
\end{array}\right.
$$

has a unique solution in $X$. Moreover, this solution can be expressed as

$$
\begin{aligned}
& u(t)=A+B t+\frac{C t^{2}}{2}+\sum_{k: t>t_{k}} \\
& \times\left[\begin{array}{c}
I_{0, k}\left(t_{k}, u\left(t_{k}\right), u^{\prime}\left(t_{k}\right), u^{\prime \prime}\left(t_{k}\right)\right)+I_{1, k}\left(t_{k}, u\left(t_{k}\right), u^{\prime}\left(t_{k}\right), u^{\prime \prime}\left(t_{k}\right)\right)\left(t-t_{k}\right) \\
+I_{2 k}\left(t_{k}, u\left(t_{k}\right), u^{\prime}\left(t_{k}\right), u^{\prime \prime}\left(t_{k}\right)\right) \frac{\left(t-t_{k}\right)^{2}}{2}
\end{array}\right] \\
& -\frac{t^{2}}{2} \sum_{k=1}^{+\infty} I_{2 k}\left(t_{k}, u\left(t_{k}\right), u^{\prime}\left(t_{k}\right), u^{\prime \prime}\left(t_{k}\right)\right)-\int_{0}^{+\infty} G(t, s) e(s) d s
\end{aligned}
$$

where $G(t, s)$ is the Green function of the homogeneous problem associated to (2.1), given by

$$
G(t, s)= \begin{cases}\frac{1}{2} s^{2}-s t, & 0 \leq s \leq t \\ -\frac{1}{2} t^{2}, & t \leq s \leq+\infty .\end{cases}
$$

The proof follows from standard integrations and usual arguments and is omitted.

The following theorem, to be used forward, gives a general criterion for relative compactness: 
Theorem 1. ( [8]) Let $M \subset C_{\infty}=\left\{x \in C[0,+\infty): \lim _{t \rightarrow+\infty} x(t)\right.$ exists $\}$. Then $M$ is relatively compact if the following conditions hold:

1. All functions in $M$ are uniformly bounded;

2. All functions in $M$ are equicontinuous on any compact interval of $[0,+\infty)$;

3. All functions in $M$ are equiconvergent at infinity, that is, for any given $\epsilon>0$, there exists a $t_{\epsilon}$ such that $|x(t)-x(+\infty)|<\epsilon$, for all $t>t_{\epsilon}$ and $x \in M$.

\section{Main result}

In this section we prove the existence of at least one solution for the problem (1.1)-(1.3), applying lower and upper solutions method. Moreover, some data on its behavior and variation are given.

First we define lower and upper functions for impulsive problems.

Definition 3. Given $A, B, C \in \mathbb{R}$, a function $\alpha \in X$ is a lower solution of problem (1.1)-(1.3) if

$$
\left\{\begin{array}{l}
\alpha^{\prime \prime \prime}(t) \geq f\left(t, \alpha(t), \alpha^{\prime}(t), \alpha^{\prime \prime}(t)\right), t \in[0,+\infty) \\
\alpha(0) \leq A, \quad \alpha^{\prime}(0) \geq B, \quad \alpha^{\prime \prime}(+\infty) \leq C \\
\Delta \alpha\left(t_{k}\right) \leq I_{0 k}\left(t_{k}, \alpha\left(t_{k}\right), \alpha^{\prime}\left(t_{k}\right), \alpha^{\prime \prime}\left(t_{k}\right)\right) \\
\Delta \alpha^{\prime}\left(t_{k}\right)>I_{1 k}\left(t_{k}, \alpha\left(t_{k}\right), \alpha^{\prime}\left(t_{k}\right), \alpha^{\prime \prime}\left(t_{k}\right)\right) \\
\Delta \alpha^{\prime \prime}\left(t_{k}\right)>I_{2 k}\left(t_{k}, \alpha\left(t_{k}\right), \alpha^{\prime}\left(t_{k}\right), \alpha^{\prime \prime}\left(t_{k}\right)\right)
\end{array}\right.
$$

with $k \in \mathbb{N}$.

A function $\beta \in X$ is an upper solution if it verifies the reversed inequalities.

Forward, the following assumption will play a key role:

(A) There is $\xi>0$ such that

$$
\xi \geq \max \left\{\begin{array}{c}
\|\alpha\|_{0},\|\beta\|_{0},\left\|\alpha^{\prime}\right\|_{1},\left\|\beta^{\prime}\right\|_{1},\left\|\alpha^{\prime \prime}\right\|_{2},\left\|\beta^{\prime \prime}\right\|_{2}, \\
|A|+\frac{|B|+|C|}{2}+\sum_{k=1}^{+\infty} \lambda_{0 k \xi}+\sum_{k=1}^{+\infty} \lambda_{1 k \xi}+\sum_{k=1}^{+\infty} \lambda_{2 k \xi} \\
+M_{0}\left(\frac{\pi}{2}+\int_{0}^{+\infty} \psi_{\xi}(s) d s\right), \\
|B|+|C|+\sum_{k=1}^{+\infty} \lambda_{1 k \xi}+2 \sum_{k=1}^{+\infty} \lambda_{2 k \xi} \\
+M_{1}\left(\frac{\pi}{2}+\int_{0}^{+\infty} \psi_{\xi}(s) d s\right), \\
\frac{|C|}{2}+\sum_{k=1}^{+\infty} \lambda_{2 k \xi}+\frac{1}{2} \int_{0}^{+\infty} \psi_{\xi}(s) d s+\frac{\pi}{4}
\end{array}\right\}
$$

where, for Catathéodory sequences $I_{i k}:[0,+\infty) \times \mathbb{R}^{3} \rightarrow \mathbb{R}, i=0,1,2$, $k \in \mathbb{N}$,

$$
\left|I_{i k}\left(t_{k}, y_{0}, y_{1}, y_{2}\right)\right| \leq \sum_{k=1}^{+\infty} \lambda_{i k \xi}<+\infty
$$

when

$$
\begin{gathered}
\left|y_{0}\right|<\xi\left(1+t^{2}\right),\left|y_{1}\right|<\xi(1+t),\left|y_{2}\right|<2 \xi, \text { for } t \in[0,+\infty), \\
M_{0}:=\sup _{t \in[0,+\infty[} \frac{|G(t, s)|}{1+t^{2}}, M_{1}:=\sup _{t \in[0,+\infty[} \frac{\left|\frac{\partial G}{\partial t}(t, s)\right|}{1+t},
\end{gathered}
$$


for $s \in[0,+\infty)$, and

$$
|f(t, x, y, z)| \leq \psi_{\xi}(t), \text { a.e. } t \in[0,+\infty),
$$

when $\max \left\{\|x\|_{0},\|y\|_{1},\|z\|_{2}\right\}<\xi$.

The existence and localization result is given by next theorem.

Theorem 2. Consider $A, B, C \in \mathbb{R}$. Assume that there are $\alpha$ and $\beta$ lower and upper solutions of problem (1.1)-(1.3) such that

$$
\alpha^{\prime \prime}(t) \leq \beta^{\prime \prime}(t), \forall t \in[0,+\infty) .
$$

Let $f:[0,+\infty] \times \mathbb{R}^{4} \rightarrow \mathbb{R}$ be a $L^{1}-$ Carathéodory function with

$$
f\left(t, \alpha(t), \alpha^{\prime}(t), y_{2}\right) \geq f\left(t, y_{0}, y_{1}, y_{2}\right) \geq f\left(t, \beta(t), \beta^{\prime}(t), y_{2}\right)
$$

for $t \in[0,+\infty], \alpha(t) \leq y_{0} \leq \beta(t), \alpha^{\prime}(t) \leq y_{1} \leq \beta^{\prime}(t)$, and $y_{2} \in \mathbb{R}$.

Assume that $I_{i k}:[0,+\infty) \times \mathbb{R}^{3} \rightarrow \mathbb{R}$ are Catathéodory sequences, for $i=0,1,2$, $k \in \mathbb{N}$, such that

$$
\begin{aligned}
& I_{0 k}\left(t_{k}, \alpha\left(t_{k}\right), \alpha^{\prime}\left(t_{k}\right), \alpha^{\prime \prime}\left(t_{k}\right)\right) \leq I_{0 k}\left(t_{k}, y_{0}, y_{1}, y_{2}\right) \leq I_{0 k}\left(t_{k}, \beta\left(t_{k}\right), \beta^{\prime}\left(t_{k}\right), \beta^{\prime \prime}\left(t_{k}\right)\right), \\
& \text { for } \alpha^{(i)}\left(t_{k}\right) \leq y_{i} \leq \beta^{(i)}\left(t_{k}\right), i=0,1,2, \\
& \left.I_{1 k}\left(t_{k}, \alpha\left(t_{k}\right), \alpha^{\prime}\left(t_{k}\right), \alpha^{\prime \prime}\left(t_{k}\right)\right) \leq I_{1 k}\left(t_{k}, y_{0}, y_{1}, y_{2}\right)\right) \leq I_{1 k}\left(t_{k}, \beta\left(t_{k}\right), \beta^{\prime}\left(t_{k}\right), \beta^{\prime \prime}\left(t_{k}\right)\right), \\
& \text { for } \alpha^{(i)}\left(t_{k}\right) \leq y_{i} \leq \beta^{(i)}\left(t_{k}\right), i=0,1,2, \\
& \left.I_{2 k}\left(t_{k}, \beta\left(t_{k}\right), \beta^{\prime}\left(t_{k}\right), y_{2}\right) \leq I_{2 k}\left(t_{k}, y_{0}, y_{1}, y_{2}\right)\right) \leq I_{2 k}\left(t_{k}, \alpha\left(t_{k}\right), \alpha^{\prime}\left(t_{k}\right), y_{2}\right) \\
& \text { for } \alpha^{(i)}\left(t_{k}\right) \leq y_{i} \leq \beta^{(i)}\left(t_{k}\right), i=0,1, y_{2} \in \mathbb{R} .
\end{aligned}
$$

If there is $\xi>0$ such that assumption (A) holds, then there is at least $u(t) \in X$, a solution of (1.1)-(1.3), such that

$$
\alpha^{(i)}(t) \leq u^{(i)}(t) \leq \beta^{(i)}(t), \forall t \in[0,+\infty), i=0,1,2 .
$$

Proof. Let $\alpha, \beta \in X$ be, respectively, lower and upper solutions of (1.1)(1.3) verifying (3.1). Notice that the relations $\alpha(t) \leq \beta(t)$ and $\alpha^{\prime}(t) \leq \beta^{\prime}(t)$, $\forall t \in[0,+\infty)$, are obtained by integration from (3.1) and the boundary conditions (1.2). Consider the modified and perturbed problem composed of the differential equation

$$
\begin{aligned}
u^{\prime \prime \prime}(t)= & f\left(t, \delta_{0}(t, u(t)), \delta_{1}\left(t, u^{\prime}(t)\right), \delta_{2}\left(t, u^{\prime \prime}(t)\right)\right) \\
& +\frac{1}{1+t^{2}} \frac{u^{\prime \prime}(t)-\delta_{2}\left(t, u^{\prime \prime}(t)\right)}{1+\left|u^{\prime \prime}(t)-\delta_{2}\left(t, u^{\prime \prime}(t)\right)\right|},
\end{aligned}
$$

for $t \in[0,+\infty)$, where the functions $\delta_{j}:[0,+\infty) \times \mathbb{R} \rightarrow \mathbb{R}, j=0,1,2$ are given by

$$
\delta_{j}\left(t, u^{(j)}(t)\right)= \begin{cases}\beta^{(j)}(t), & u^{(j)}(t)>\beta^{(j)}(t), \\ u^{(j)}(t), & \alpha^{(j)}(t) \leq u^{(j)}(t) \leq \beta^{(j)}(t), \\ \alpha^{(j)}(t), & u^{(j)}(t)<\alpha^{(j)}(t),\end{cases}
$$


the boundary conditions (1.2) and the truncated impulsive effects

$$
\Delta u^{(j)}\left(t_{k}\right)=I_{j, k}\left(t_{k}, \delta_{0}\left(t_{k}, u\left(t_{k}\right)\right), \delta_{1}\left(t_{k}, u^{\prime}\left(t_{k}\right)\right), \delta_{2}\left(t_{k}, u^{\prime \prime}\left(t_{k}\right)\right)\right), j=0,1,2 .
$$

For clearity we divide the proof into claims.

CLAIM 1: Problem (3.6),(1.2), (3.7) has at least one solution.

Define the operator $\mathcal{T}: X \rightarrow X$ by

$$
\begin{aligned}
& \mathcal{T} u(t)=A+B t+\frac{C t^{2}}{2}+\sum_{k: t>t_{k}} \\
& \times\left[\begin{array}{c}
I_{0, k}\left(t_{k}, u\left(t_{k}\right), u^{\prime}\left(t_{k}\right), u^{\prime \prime}\left(t_{k}\right)\right)+I_{1, k}\left(t_{k}, u\left(t_{k}\right), u^{\prime}\left(t_{k}\right), u^{\prime \prime}\left(t_{k}\right)\right)\left(t-t_{k}\right) \\
+I_{2 k}\left(t_{k}, u\left(t_{k}\right), u^{\prime}\left(t_{k}\right), u^{\prime \prime}\left(t_{k}\right)\right) \frac{\left(t-t_{k}\right)^{2}}{2}
\end{array}\right] \\
& -\frac{t^{2}}{2} \sum_{k=1}^{+\infty} I_{2 k}\left(t_{k}, u\left(t_{k}\right), u^{\prime}\left(t_{k}\right), u^{\prime \prime}\left(t_{k}\right)\right)-\int_{0}^{+\infty} G(t, s) F(u(s)) d s
\end{aligned}
$$

with $G(t, s)$ given by $(2.2)$, and

$$
\begin{aligned}
F(u(s)):= & f\left(s, \delta_{0}(s, u(s)), \delta_{1}\left(s, u^{\prime}(s)\right), \delta_{2}\left(s, u^{\prime \prime}(s)\right)\right) \\
& +\frac{1}{1+t^{2}} \frac{u^{\prime \prime}(s)-\delta_{2}\left(t, u^{\prime \prime}(s)\right)}{1+\left|u^{\prime \prime}(s)-\delta_{2}\left(s, u^{\prime \prime}(s)\right)\right|} .
\end{aligned}
$$

By Lemma 1, the fixed points of $\mathcal{T}$ are solutions of the problem (3.6), (1.2) and (3.7). So it is enough to prove that $\mathcal{T}$ has a fixed point.

For convenience we denote

$$
I_{i, k}:=I_{i, k}\left(t_{k}, u\left(t_{k}\right), u^{\prime}\left(t_{k}\right), u^{\prime \prime}\left(t_{k}\right)\right) \text {, for } i=0,1,2 .
$$

(1) $\mathcal{T}: X \rightarrow X$ is well defined.

Take

$$
\rho>\max \left\{\|\alpha\|_{0},\|\beta\|_{0},\left\|\alpha^{\prime}\right\|_{1},\left\|\beta^{\prime}\right\|_{1},\left\|\alpha^{\prime \prime}\right\|_{2},\left\|\beta^{\prime \prime}\right\|_{2}\right\} .
$$

As $f$ is an $L^{1}$-Carathéodory function, by Definition 1 , for $u \in X$ with $\|u\|<\rho$,

$$
\int_{0}^{+\infty}|F(u(s))| d s \leq \int_{0}^{+\infty}\left(\psi_{\rho}(s)+\frac{1}{1+t^{2}}\right) d s \leq \int_{0}^{+\infty} \psi_{\rho}(s) d s+\frac{\pi}{2}<+\infty,
$$

and so $F(u(s)) \in L^{1}([0,+\infty[)$.

By Lebesgue Dominated Theorem and Definition 2,

$$
\lim _{t \rightarrow+\infty} \frac{|\mathcal{T} u(t)|}{1+t^{2}} \leq \frac{|C|}{2}+\sum_{k=1}^{+\infty}\left|I_{2 k}\right| \leq \frac{|C|}{2}+\sum_{k=1}^{+\infty} \lambda_{2 k \rho}<+\infty
$$

Analogously,

$$
\lim _{t \rightarrow+\infty} \frac{\left|\mathcal{T} u^{\prime}(t)\right|}{1+t} \leq|C|+2 \sum_{k=1}^{+\infty}\left|I_{2 k}\right| \leq|C|+2 \sum_{k=1}^{+\infty} \lambda_{2 k \rho}<+\infty
$$


and

$$
\lim _{t \rightarrow+\infty} \frac{\left|(\mathcal{T} u)^{\prime \prime}(t)\right|}{2} \leq \frac{1}{2}\left(|C|+2 \sum_{k=1}^{+\infty} \lambda_{2 k \rho}+\int_{0}^{+\infty} \psi_{\rho}(s) d s\right)<+\infty .
$$

Therefore, $\mathcal{T} u \in X$.

(2) $\mathcal{T}$ is continuous.

For any convergent sequence $u_{n} \rightarrow u$ in $X$, there exists $r_{1}>0$ such that, for $\left\|u_{n}\right\|<r_{1}$, we have

$$
\begin{aligned}
& \left\|\mathcal{T} u_{n}-\mathcal{T} u\right\|=\max \left\{\left\|\mathcal{T} u_{n}-\mathcal{T} u\right\|_{0},\left\|\left(\mathcal{T} u_{n}\right)^{\prime}-(\mathcal{T} u)^{\prime}\right\|_{1},\left\|\left(\mathcal{T} u_{n}\right)^{\prime \prime}-(\mathcal{T} u)^{\prime \prime}\right\|_{2}\right\} \\
& \quad \leq \int_{0}^{+\infty} \max \left\{M_{0}, M_{1}\right\}\left|F\left(u_{n}(s)\right)-F(u(s))\right| d s \\
& \quad \leq \int_{0}^{+\infty}\left|F\left(u_{n}(s)\right)-F(u(s))\right| d s \longrightarrow 0, \quad n \rightarrow+\infty .
\end{aligned}
$$

(3) $\mathcal{T}$ is compact.

Let $D \subset X$ be any bounded subset. Therefore there is $R>0$ such that $\|u\|<R, \forall u \in D$. Then,

$$
\begin{aligned}
& \|\mathcal{T} u\|_{0}=\sup _{t \in[0,+\infty[} \frac{|\mathcal{T} u(t)|}{1+t^{2}} \leq|A|+\frac{|B|+|C|}{2}+\sum_{k=1}^{+\infty}\left|I_{0 k}\right| \\
& +\sum_{k=1}^{+\infty}\left|I_{1 k}\right|+\sum_{k=1}^{+\infty}\left|I_{2 k}\right|+\int_{0}^{+\infty} \sup _{t \in[0,+\infty[} \frac{|G(t, s)|}{1+t^{2}} \psi_{R}(s) d s \\
& \leq|A|+\frac{|B|+|C|}{2}+\sum_{k=1}^{+\infty} \lambda_{0 k R}+\sum_{k=1}^{+\infty} \lambda_{1 k R}+\sum_{k=1}^{+\infty} \lambda_{2 k R} \\
& +M_{0}\left(\frac{\pi}{2}+\int_{0}^{+\infty} \psi_{R}(s) d s\right)<+\infty \\
& \|\mathcal{T} u\|_{1}=\sup _{t \in[0,+\infty[} \frac{\left|(\mathcal{T} u(t))^{\prime}\right|}{1+t} \\
& \leq|B|+|C|+\sup _{t \in[0,+\infty[} \frac{1}{1+t} \sum_{k: t>t_{k}}\left|I_{1 k}\right|+\sup _{t \in\left[0,+\infty\left[\left[_{k}: t>t_{k}\right.\right.\right.}\left|I_{2 k}\right| \frac{t-t_{k}}{1+t} \\
& +\sup _{t \in[0,+\infty[} \frac{t}{1+t} \sum_{k=1}^{+\infty}\left|I_{2 k}\right|+\int_{0}^{+\infty} M_{1}|F(u(s))| d s \\
& \leq|B|+|C|+\sum_{k=1}^{+\infty} \lambda_{1 k R}+2 \sum_{k=1}^{+\infty} \lambda_{2 k R}+M_{1}\left(\frac{\pi}{2}+\int_{0}^{+\infty} \psi_{p}(s) d s\right)<+\infty,
\end{aligned}
$$

and

$$
\|\mathcal{T} u\|_{2}=\sup _{t \in[0,+\infty[} \frac{\left|(\mathcal{T} u(t))^{\prime \prime}\right|}{2} \leq \frac{|C|}{2}+\sum_{k=1}^{+\infty} \lambda_{2 k R}+\frac{1}{2} \int_{0}^{+\infty} \psi_{R}(s) d s+\frac{\pi}{4}<+\infty .
$$


So $\mathcal{T}$ is uniformly bounded. Moreover, $\mathcal{T}$ is equicontinuous on each interval $\left(t_{k}, t_{k+1}\right]$. To see this, let $t_{1}, t_{2} \in\left(t_{k}, t_{k+1}\right]$, with $t_{1}<t_{2} ;$ as $t_{1} \rightarrow t_{2}$,

$$
\begin{aligned}
& \left|\frac{\mathcal{T} u\left(t_{1}\right)}{1+t_{1}^{2}}-\frac{\mathcal{T} u\left(t_{2}\right)}{1+t_{2}^{2}}\right| \leq\left|\frac{B t_{1}+\frac{C}{2} t_{1}^{2}}{1+t_{1}^{2}}-\frac{B t_{2}+\frac{C}{2} t_{2}^{2}}{1+t_{2}^{2}}\right| \\
& +\left|\begin{array}{l}
\sum_{k}: t_{1}>t_{k} \\
-\sum_{k: t_{2}>t_{k}}\left[I_{0 k}+I_{1 k}\left(t_{1}-t_{k}\right)+I_{2 k} \frac{\left(t_{1}-t_{k}\right)^{2}}{2}\right]
\end{array}\right|+\frac{1}{2}\left|t_{1}^{2}-t_{2}^{2}\right| \sum_{k=1}^{+\infty}\left|I_{2 k}\right| \\
& \quad+\int_{0}^{+\infty}\left|\frac{G\left(t_{1}, s\right)}{1+t_{1}^{2}}-\frac{G\left(t_{2}, s\right)}{1+t_{2}^{2}}\right|\left(\psi_{R}(s)+1\right) d s \longrightarrow 0
\end{aligned}
$$

and

$$
\begin{aligned}
& \left|\frac{(\mathcal{T} u)^{\prime}\left(t_{1}\right)}{1+t_{1}}-\frac{(\mathcal{T} u)^{\prime}\left(t_{2}\right)}{1+t_{2}}\right| \leq\left|\frac{C t_{1}}{1+t_{1}}-\frac{C t_{2}}{1+t_{2}}\right|+\left|t_{1}-t_{2}\right| \sum_{k=1}^{+\infty}\left|I_{2 k}\right| \\
& \quad+\left|\sum_{k: t_{1}>t_{k}}\left[I_{1 k}+I_{2 k}\left(t_{1}-t_{k}\right)\right]-\sum_{k: t_{2}>t_{k}}\left[I_{1 k}+I_{2 k}\left(t_{2}-t_{k}\right)\right]\right| \\
& \quad+\int_{0}^{+\infty}\left|\frac{\frac{\partial G}{\partial t}\left(t_{1}, s\right)}{1+t_{1}^{2}}-\frac{\frac{\partial G}{\partial t}\left(t_{2}, s\right)}{1+t_{2}^{2}}\right|\left(\psi_{R}(s)+1\right) d s \longrightarrow 0 .
\end{aligned}
$$

The function $\frac{\partial^{2} G}{\partial t^{2}}(t, s)$ is not continuous for $s=t$ but the jump is controlled by 1 . Then,

$$
\begin{aligned}
& \left|\frac{(\mathcal{T} u)^{\prime \prime}\left(t_{1}\right)}{2}-\frac{(\mathcal{T} u)^{\prime \prime}\left(t_{2}\right)}{2}\right| \leq \frac{1}{2} \sum_{k: t_{1}<t_{k}<t_{2}}\left|I_{2 k}\right| \\
& +\left|\int_{t_{1}}^{+\infty} F(u(s)) d s-\int_{t_{2}}^{+\infty} F(u(s)) d s\right| \\
& \leq \frac{1}{2} \sum_{k: t_{1}<t_{k}<t_{2}}\left|I_{2 k}\right|+\int_{t_{1}}^{t_{2}}\left(\psi_{R}(s)+1\right) d s \longrightarrow 0 \text { as } t_{1} \rightarrow t_{2} .
\end{aligned}
$$

To prove that $\mathcal{T} D$ is equiconvergent at infinity we apply, as $t \rightarrow+\infty$,

$$
\begin{aligned}
& \left|\frac{\mathcal{T} u(t)}{1+t^{2}}-\lim _{t \rightarrow+\infty} \frac{\mathcal{T} u(t)}{1+t^{2}}\right| \leq\left|\frac{A+B t}{1+t^{2}}+\frac{C t^{2}}{2+2 t^{2}}-\frac{C}{2}\right| \\
& +\frac{1}{1+t^{2}} \mid \sum_{k: t>t_{k}}\left[I_{0 k}+I_{1 k}\left(t_{1}-t_{k}\right)+I_{2 k} \frac{\left(t_{1}-t_{k}\right)^{2}}{2}\right] \\
& +\mid \frac{1}{2} \sum_{k=1}^{+\infty} I_{2 k}
\end{aligned}
$$




$$
\begin{aligned}
& \left|\frac{(\mathcal{T} u)^{\prime}(t)}{1+t}-\lim _{t \rightarrow+\infty} \frac{(\mathcal{T} u)^{\prime}(t)}{1+t}\right| \leq\left|\frac{B+C t}{1+t}-C\right| \\
& \quad+\frac{1}{1+t}\left|\sum_{k: t>t_{k}}\left[I_{1 k}+I_{2 k}\left(t_{1}-t_{k}\right)\right]-\sum_{k=1}^{+\infty} I_{2 k}\right| \\
& \quad+\left|\frac{t}{1+t} \sum_{k=1}^{+\infty}\right| I_{2 k}\left|-\sum_{k=1}^{+\infty}\right| I_{2 k}|| \\
& \quad+\int_{0}^{+\infty}\left|\frac{\frac{\partial G}{\partial t}(t, s)}{1+t}+1\right|\left(\psi_{R}(s)+1\right) d s \longrightarrow 0, \\
& \left|\frac{(\mathcal{T} u)^{\prime \prime}(t)}{2} \lim _{t \rightarrow+\infty} \frac{(\mathcal{T} u)^{\prime \prime}(t)}{2}\right| \leq \frac{1}{2}\left|\sum_{k: t>t_{k}} I_{2 k}-\sum_{k=1}^{+\infty} I_{2 k}\right| \\
& +\int_{0}^{+\infty}\left|\frac{\partial^{2} G}{\partial t^{2}}(t, s)+1\right|\left(\psi_{R}(s)+1\right) d s \longrightarrow 0, \text { as } t \rightarrow+\infty .
\end{aligned}
$$

Finally, to prove that $\mathcal{T} D$ is equiconvergent at the impulsive moments we apply, as $t \rightarrow t_{i}^{+}$, for $i \in \mathbb{N}$,

$$
\begin{aligned}
& \left|\frac{\mathcal{T} u(t)}{1+t^{2}}-\lim _{t \rightarrow t_{i}^{+}} \frac{\mathcal{T} u(t)}{1+t^{2}}\right| \leq\left|\frac{B t+\frac{C}{2} t^{2}}{1+t^{2}}-\frac{B t_{i}+\frac{C}{2} t_{i}^{2}}{1+t_{i}^{2}}\right| \\
& +\mid \begin{array}{c}
\frac{1}{1+t^{2}} \sum_{k: t>t_{k}}\left(I_{0 k}+I_{1 k}\left(t-t_{k}\right)+I_{2 k} \frac{\left(t-t_{k}\right)^{2}}{2}\right) \\
-\frac{1}{1+t_{i}^{2}} \sum_{k: t_{i}^{+}>t_{k}}\left(I_{0 k}+I_{1 k}\left(t_{i}^{+}-t_{k}\right)+I_{2 k} \frac{\left(t_{i}^{+}-t_{k}\right)^{2}}{2}\right)
\end{array} \\
& +\left|\left(-\frac{t^{2}}{1+t^{2}}+\frac{t_{i}^{2}}{1+t_{i}^{2}}\right) \sum_{k=1}^{+\infty} I_{2 k}\right| \\
& \left.+\int_{0}^{+\infty}\left|\frac{G(t, s)}{1+t^{2}}-\frac{G\left(t_{i}^{+}, s\right)}{1+t_{i}^{2}}\right|\left(\psi_{R}(s)+1\right)\right) d s \longrightarrow 0,
\end{aligned}
$$

uniformly on $u \in D$, as $t \longrightarrow t_{i}^{+}$,

$$
\begin{aligned}
& \left|\frac{(\mathcal{T} u)^{\prime}(t)}{1+t}-\lim _{t \rightarrow t_{i}^{+}} \frac{(\mathcal{T} u)^{\prime}(t)}{1+t}\right| \leq\left|\frac{C t}{1+t}-\frac{C t_{i}}{1+t_{i}}\right| \\
& +\left|\frac{1}{1+t} \sum_{k: t>t_{k}}\left[I_{1 k}+I_{2 k}\left(t-t_{k}\right)\right]-\frac{1}{1+t_{i}} \sum_{t_{i}^{+}>t_{k}}\left[I_{1 k}+I_{2 k}\left(t_{i}-t_{k}\right)\right]\right| \\
& +\left|\left(-\frac{t}{1+t}+\frac{t_{i}}{1+t_{i}}\right) \sum_{k=1}^{+\infty} I_{2 k}\right| \\
& \left.+\int_{0}^{+\infty}\left|\frac{\frac{\partial}{\partial t} G(t, s)}{1+t^{2}}-\frac{\frac{\partial}{\partial t} G\left(t_{i}^{+}, s\right)}{1+t_{i}^{2}}\right|\left(\psi_{R}(s)+1\right)\right) d s \longrightarrow 0, \text { as } t \longrightarrow t_{i}^{+}, \\
& \left|\frac{(\mathcal{T} u)^{\prime \prime}(t)}{2}-\lim _{t \rightarrow t_{i}^{+}} \frac{(\mathcal{T} u)^{\prime \prime}(t)}{2}\right| \leq\left|\frac{1}{2} \sum_{k: t>t_{k}} I_{2 k}-\frac{1}{2} \sum_{k: t_{i}^{+}>t_{k}} I_{2 k}\right|
\end{aligned}
$$




$$
\begin{aligned}
& +\frac{1}{2}\left|\int_{t}^{+\infty} F(u(s)) d s-\int_{t_{i}}^{+\infty} F(u(s)) d s\right| \\
\leq & \frac{1}{2}\left|\sum_{k: t>t_{k}} I_{2 k}-\sum_{k: t_{i}^{+}>t_{k}} I_{2 k}\right|+\frac{1}{2}\left|\int_{t_{i}}^{t}\left(\psi_{R}(s)+1\right) d s\right| \longrightarrow 0,
\end{aligned}
$$

uniformly for $u \in D$, as $t \longrightarrow t_{i}^{+}$.

So, by Theorem $1, \mathcal{T} D$ is relatively compact. To apply Schauder's Fixed Point Theorem, we need to show that $\mathcal{T}: D \rightarrow D$.

CLAIM 2: For some nonempty, closed, bounded and convex subset $D \subset X$, $\mathcal{T} D \subseteq D$. that

By assumption $(A)$ in Step (3) of the previous Claim 1, take $R>0$ such

$$
R \geq \max \left\{\begin{array}{c}
\rho,|A|+\frac{|B|+|C|}{2}+\sum_{k=1}^{+\infty} \lambda_{0 k R}+\sum_{k=1}^{+\infty} \lambda_{1 k R}+\sum_{k=1}^{+\infty} \lambda_{2 k R} \\
+M_{0}\left(\frac{\pi}{2}+\int_{0}^{+\infty} \psi_{R}(s) d s\right), \\
|B|+|C|+\sum_{k=1}^{+\infty} \lambda_{1 k R}+2 \sum_{k=1}^{+\infty} \lambda_{2 k R} \\
+M_{1}\left(\frac{\pi}{2}+\int_{0}^{+\infty} \psi_{R}(s) d s\right), \\
\frac{|C|}{2}+\sum_{k=1}^{+\infty} \lambda_{2 k R}+\frac{1}{2} \int_{0}^{+\infty} \psi_{R}(s) d s+\frac{\pi}{4}
\end{array}\right\},
$$

with $\rho$ given by (3.8).

From the calculations in Claim 1, for every $u \in D$ such that $\|u\|<R$, we have $\mathcal{T} D \subseteq D$. Hence $\mathcal{T}$ is completely continuous, by Schauder's Fixed Point Theorem, $\mathcal{T}$ has at least one fixed point $u \in X$.

From Lemma 1, (3.6) and (3.7), this fixed point will be a solution of the problem (1.1)-(1.3) if

$$
\alpha^{(i)}(t) \leq u^{(i)}(t) \leq \beta^{(i)}(t), i=0,1,2, \forall t \in[0,+\infty) .
$$

CLAIM 3: Every solution of problem (3.6), (2), (3.7), satisfies

$$
\alpha^{(i)}(t) \leq u^{(i)}(t) \leq \beta^{(i)}(t), i=0,1,2, \forall t \in[0,+\infty) .
$$

Let $u$ be a solution of problem (3.6), (2), (3.7). Suppose for a contradiction that there is $u^{\prime \prime}(t)<\alpha^{\prime \prime}(t)$, and define

$$
\inf _{t \in[0,+\infty[} u^{\prime \prime}(t)-\alpha^{\prime \prime}(t):=u^{\prime \prime}\left(t_{*}\right)-\alpha^{\prime \prime}\left(t_{*}\right)<0 .
$$

Note that $t_{*} \neq+\infty$, as by (2) and Definition 3, $u^{\prime \prime}(+\infty)-\alpha^{\prime \prime}(+\infty) \geq 0$.

If $t_{*}=0$, the following contradiction holds, by (3.2) and Definition 3:

$$
\begin{aligned}
0 & \leq u^{\prime \prime \prime}(0)-\alpha^{\prime \prime \prime}(0)=f\left(0, \delta_{0}(0, u(0)), \delta_{1}\left(0, u^{\prime}(0)\right), \delta_{2}\left(0, u^{\prime \prime}(0)\right)\right) \\
+ & \frac{u^{\prime \prime}(0)-\alpha^{\prime \prime}(0)}{1+\left|u^{\prime \prime}(0)-\alpha^{\prime \prime}(0)\right|}-\alpha^{\prime \prime \prime}(0)<f\left(0, \delta_{0}(0, u(0)), \delta_{1}\left(0, u^{\prime}(0)\right), \delta_{2}\left(0, u^{\prime \prime}(0)\right)\right) \\
& \quad-\alpha^{\prime \prime \prime}(0) \leq f\left(0, \alpha(0), \alpha^{\prime}(0), \alpha^{\prime \prime}(0)\right)-\alpha^{\prime \prime \prime}(0) \leq 0 .
\end{aligned}
$$


Therefore $t_{*} \neq 0$.

Consider now that $t_{*}$ is between two consecutive impulses. That is, there is a $p \in \mathbb{N}$ such that $t_{*} \in\left(t_{p}, t_{p+1}\right)$. Then,

$$
u^{\prime \prime \prime}\left(t_{*}\right)=\alpha^{\prime \prime \prime}\left(t_{*}\right), u^{\prime \prime}\left(t_{*}\right)-\alpha^{\prime \prime}\left(t_{*}\right)<0
$$

and we have, by (3.2) and Definition 3, the contradiction

$$
\begin{aligned}
& 0=u^{\prime \prime \prime}\left(t_{*}\right)-\alpha^{\prime \prime \prime}\left(t_{*}\right)=f\left(t_{*}, \delta_{0}\left(t_{*}, u\left(t_{*}\right)\right), \delta_{1}\left(t_{*}, u^{\prime}\left(t_{*}\right)\right), u^{\prime \prime}\left(t_{*}\right)\right) \\
& +\frac{1}{1+t_{*}^{2}} \frac{u^{\prime \prime}\left(t_{*}\right)-\delta_{2}\left(t_{*}, u\left(t_{*}\right)\right)}{\left|u^{\prime \prime}\left(t_{*}\right)-\delta_{2}\left(t_{*}, u\left(t_{*}\right)\right)\right|+1}-\alpha^{\prime \prime \prime}\left(t_{*}\right) \\
& \leq f\left(t_{*}, \alpha\left(t_{*}\right), \alpha^{\prime}\left(t_{*}\right), \alpha^{\prime \prime}\left(t_{*}\right)\right)+\frac{1}{1+t_{*}^{2}} \frac{u^{\prime \prime}\left(t_{*}\right)-\alpha^{\prime \prime}\left(t_{*}\right)}{\left|u^{\prime \prime}\left(t_{*}\right)-\alpha^{\prime \prime}\left(t_{*}\right)\right|+1}-\alpha^{\prime \prime \prime}\left(t_{*}\right) \\
& <f\left(t_{*}, \alpha\left(t_{*}\right), \alpha^{\prime}\left(t_{*}\right), \alpha^{\prime \prime}\left(t_{*}\right)\right)-\alpha^{\prime \prime}\left(t_{*}\right) \leq 0 \text {. }
\end{aligned}
$$

Assume now that the infimum is attained at an impulsive moment. So, we have two cases: $t_{*}=t_{q}^{-}$or $t_{*}=t_{q}^{+}$. Firstly, consider that there is $q \in \mathbb{N}$ where

$$
\min _{t \in[0,+\infty)}\left(u^{\prime \prime}(t)-\alpha^{\prime \prime}(t)\right):=u^{\prime \prime}\left(t_{q}\right)-\alpha^{\prime \prime}\left(t_{q}\right)<0 .
$$

Then this contradiction holds:

$$
\begin{aligned}
0 & \leq \Delta\left(u^{\prime \prime}-\alpha^{\prime \prime}\right)\left(t_{q}\right) \\
& =I_{2, q}\left(t_{q}, \delta_{0}\left(t_{q}, u\left(t_{q}\right)\right), \delta_{1}\left(t_{q}, u^{\prime}\left(t_{q}\right)\right), \delta_{2}\left(t_{q}, u^{\prime \prime}\left(t_{q}\right)\right)\right)-\Delta \alpha^{\prime \prime}\left(t_{q}\right) \\
& =I_{2, q}\left(t_{q}, \delta_{0}\left(t_{q}, u\left(t_{q}\right)\right), \delta_{1}\left(t_{q}, u^{\prime}\left(t_{q}\right)\right), \alpha^{\prime \prime}\left(t_{q}\right)\right)-\Delta \alpha^{\prime \prime}\left(t_{q}\right) \\
& \leq I_{2, q}\left(t_{q}, \alpha\left(t_{q}\right), \alpha^{\prime}\left(\left(t_{q}\right), \alpha^{\prime \prime}\left(t_{q}\right)\right)-\Delta \alpha^{\prime \prime}\left(t_{q}\right)<0 .\right.
\end{aligned}
$$

In the second case, assume that

$$
\inf _{t \in[0,+\infty)} u^{\prime \prime}(t)-\alpha^{\prime \prime}(t):=u^{\prime \prime}\left(t_{*}^{+}\right)-\alpha^{\prime \prime}\left(t_{*}^{+}\right)<0 .
$$

Consider $\varepsilon>0$ small enough such that

$$
\left(u^{\prime \prime}-\alpha^{\prime \prime}\right)(t)<0, u^{\prime \prime \prime}\left(t^{+}\right)-\alpha^{\prime \prime \prime}\left(t^{+}\right) \geq 0, \text { for } t \in\left(t_{q}, t_{q}+\varepsilon\right) .
$$

So, for $t \in\left(t_{q}, t_{q}+\varepsilon\right)$, a contradiction can be obtained following the same arguments as for $t_{*} \in\left(t_{p}, t_{p+1}\right)$. Therefore,

$$
\alpha^{\prime \prime}(t) \leq u^{\prime \prime}(t), \text { for } t \in[0,+\infty)
$$

By a similar technique, it can be proved that $u^{\prime \prime}(t) \leq \beta^{\prime \prime}(t)$, for $t \in(0,+\infty)$, and then

$$
\alpha^{\prime \prime}(t) \leq u^{\prime \prime}(t) \leq \beta^{\prime \prime}(t), \text { for } t \in[0,+\infty) .
$$

Integrating the first inequality of $(3.9)$ for $t \in\left[0, t_{1}\right]$, by (1.2) and Definition 3 ,

$$
\alpha^{\prime}(t) \leq u^{\prime}(t)+\alpha^{\prime}(0)-u^{\prime}(0) \leq u^{\prime}(t) .
$$


By integration in $\left(t_{1},+\infty\right),(3.5),(3.10)$ and Definition 3, we have for $t \in$ $\left(t_{1},+\infty\right)$

$$
\begin{aligned}
& \alpha^{\prime}(t) \leq u^{\prime}(t)+\alpha^{\prime}\left(t_{1}^{+}\right)-u^{\prime}\left(t_{1}^{+}\right)=u^{\prime}(t)+\alpha^{\prime}\left(t_{1}^{+}\right) \\
& \quad-I_{11}\left(t_{1}, \delta_{0}\left(t_{1}, u\left(t_{1}\right)\right), \delta_{1}\left(t_{1}, u^{\prime}\left(t_{1}\right)\right), \delta_{2}\left(t_{1}, u^{\prime \prime}\left(t_{1}\right)\right)\right)-u^{\prime}\left(t_{1}\right) \\
& \leq u^{\prime}(t)+I_{11}\left(t_{1}, \alpha\left(t_{1}\right), \alpha^{\prime}\left(t_{1}\right), \alpha^{\prime \prime}\left(t_{1}\right)\right)+\alpha^{\prime}\left(t_{1}\right) \\
& \quad-I_{11}\left(t_{1}, \delta_{0}\left(t_{1}, u\left(t_{1}\right)\right), u^{\prime}\left(t_{1}\right), u^{\prime \prime}\left(t_{1}\right)\right)-u^{\prime}\left(t_{1}\right) \\
& \leq u^{\prime}(t)+I_{11}\left(t_{1}, \alpha\left(t_{1}\right), \alpha^{\prime}\left(t_{1}\right), \alpha^{\prime \prime}\left(t_{1}\right)\right)-I_{11}\left(t_{1}, \delta_{0}\left(t_{1}, u\left(t_{1}\right)\right), u^{\prime}\left(t_{1}\right), u^{\prime \prime}\left(t_{1}\right)\right) \\
& \leq u^{\prime}(t) .
\end{aligned}
$$

Analogously, one can show that $u^{\prime}(t) \leq \beta^{\prime}(t), \forall t \in[0,+\infty)$ and, then,

$$
\alpha^{\prime}(t) \leq u^{\prime}(t) \leq \beta^{\prime}(t), \text { for } t \in[0,+\infty) .
$$

Integrating the first inequality of $(3.11)$ on $\left[0, t_{1}\right]$, we have

$$
\alpha(t) \leq u(t)-u(0)+\alpha(0) \leq u(t),
$$

and on $\left(t_{1},+\infty\right)$, by $(3.3)$ and Definition 3 ,

$$
\begin{aligned}
& \alpha(t) \leq u(t)+\alpha\left(t_{1}^{+}\right)-u\left(t_{1}^{+}\right) \leq u(t)+I_{01}\left(t_{1}, \alpha\left(t_{1}\right), \alpha^{\prime}\left(t_{1}\right), \alpha^{\prime \prime}\left(t_{1}\right)\right) \\
& \quad+\alpha\left(t_{1}\right)-I_{01}\left(t_{1}, \delta_{0}\left(t_{1}, u\left(t_{1}\right)\right), \delta_{1}\left(t_{1}, u^{\prime}\left(t_{1}\right)\right), \delta_{2}\left(t_{1}, u^{\prime \prime}\left(t_{1}\right)\right)\right)-u\left(t_{1}\right) \\
& \quad \leq u(t)+I_{01}\left(t_{1}, \alpha\left(t_{1}\right), \alpha^{\prime}\left(t_{1}\right), \alpha^{\prime \prime}\left(t_{1}\right)\right)-I_{01}\left(t_{1}, u\left(t_{1}\right), u^{\prime}\left(t_{1}\right), u^{\prime \prime}\left(t_{1}\right)\right) \leq u(t) .
\end{aligned}
$$

So, $\alpha(t) \leq u^{\prime}(t), \forall t \in[0,+\infty)$, and the remaining inequality $u(t) \leq \beta(t), \forall t \in$ $[0,+\infty)$, can be proved using the same technique.

\section{Example}

Problems of boundary layer flow over a stretching sheet, with and without heat transfer, are a topic that arouses growing interest in the literature (see, for example $[7,15,20,23])$. These papers deal with a boundary value problem of normal stagnation point flow impinging on a stretching sheet, governed by the parameter $b$ which represents the ratio of the strain rate of the stagnation flow to that of the stretching sheet. Existing numerical studies on the basic flow shows that a solution exists for all values of $b>0$.

In $[22]$, the third order differential equation

$$
f^{\prime \prime \prime}+f f^{\prime \prime}-\left(f^{\prime}\right)^{2}+b^{2}=0,
$$

together with the boundary conditions

$$
f(0)=0, f^{\prime}(0)=1, f^{\prime}(\infty)=b,
$$

is studied.

Motivated by this paper, in this application, we prove the solvability of the impulsive third order problem composed of a differential equation similar to 
(4.1), namely,

$$
u^{\prime \prime \prime}(t)=\left\{\begin{array}{c}
-\frac{0.01}{1+t^{2}}\left(\sqrt[3]{u(t)} \sqrt{\left|u^{\prime \prime}(t)\right|}+\operatorname{sgn}\left(u^{\prime}(t)\right) \sqrt{\left|u^{\prime}(t)\right|}-\operatorname{sgn}\left(u^{\prime \prime}(t)\right) b^{2}\right), \\
\text { if } 0 \leq t \leq 1, \\
-\frac{0.01}{1+t^{2}}\left(\sqrt[3]{u(t)} \sqrt{\left|u^{\prime \prime}(t)\right|}+\operatorname{sgn}\left(u^{\prime}(t)\right) \sqrt{\left|u^{\prime}(t)\right|}+10 \operatorname{sgn}\left(u^{\prime \prime}(t)\right) b^{2}\right), \\
\text { if } t>1,
\end{array}\right.
$$

with $b \in \mathbb{R} \backslash\{0\}$, where $u(t)$ represents the flow speed across a time $t$, together with the asymptotic boundary conditions

$$
u(0)=A, u^{\prime}(0)=B, u^{\prime \prime}(+\infty)=0,
$$

for $A, B \in \mathbb{R}$, and the impulsive effects with the form

$$
\begin{aligned}
\Delta u\left(t_{k}\right) & =\frac{1}{\left(t_{k}\right)^{3}}\left(\lambda_{01} \sqrt[3]{u\left(t_{k}\right)}+\lambda_{02}\left(u^{\prime}\left(t_{k}\right)\right)+\lambda_{03} \sqrt[3]{u^{\prime \prime}\left(t_{k}\right)}\right), \\
\Delta u^{\prime}\left(t_{k}\right) & =\frac{1}{\left(t_{k}\right)^{3}}\left(\lambda_{11}\left(u\left(t_{k}\right)\right)+\lambda_{12} \sqrt[3]{u^{\prime}\left(t_{k}\right)}\right), \\
\Delta u^{\prime \prime}\left(t_{k}\right) & =\frac{1}{\left(t_{k}\right)^{3}}\left(\lambda_{21} \sqrt[3]{u\left(t_{k}\right)}+\lambda_{22}\left(u^{\prime}\left(t_{k}\right)\right)+\lambda_{23}\left(u^{\prime \prime}\left(t_{k}\right)\right)+\operatorname{sgn}\left(u^{\prime \prime}\left(t_{k}\right)\right) * 5 t_{k}\right),
\end{aligned}
$$

where $\lambda_{i j} \in \mathbb{R}$, for $1=0,1,2$ and $j=1,2,3$, and $k \in \mathbb{N}$.

Note that:

1. The null function is not a solution of (4.2).

2. In (4.2), from a theoretical point of view, the parameter $b$ could be nonpositive.

3. For functions $u \in X$, the condition $u^{\prime \prime}(+\infty)=0$ implies that $u^{\prime}(+\infty)$ is finite.

4. Neither (4.2) nor (4.4) are covered by Theorem 3.1 of [29], as they are not sublinear and have different monotonicities.

5. The problem (4.2)-(4.4) is a particular case of the initial problem (1.1)(1.3), with $C=0$,

$$
\begin{gathered}
f\left(t, y_{0}, y_{1}, y_{2}\right)= \\
\left\{\begin{array}{c}
-\frac{0.01}{1+t^{2}}\left(\sqrt[3]{y_{0}} \sqrt{\left|y_{2}\right|}+\operatorname{sgn}\left(y_{1}\right) \sqrt{\left|y_{1}\right|}-\operatorname{sgn}\left(y_{2}\right) b^{2}\right), \text { if } 0 \leq t \leq 1, \\
-\frac{0.01}{1+t^{2}}\left(\sqrt[3]{y_{0}} \sqrt{\left|y_{2}\right|}+\operatorname{sgn}\left(y_{1}\right) \sqrt{\left|y_{1}\right|}+10 \operatorname{sgn}\left(y_{2}\right) b^{2}\right), \text { if } t>1,
\end{array}\right. \\
I_{0,1}\left(t_{k}, w_{0}, w_{1}, w_{2}\right)=\frac{1}{\left(t_{k}\right)^{3}}\left(\lambda_{01} \sqrt[3]{w_{0}}+\lambda_{02}\left(w_{1}\right)+\lambda_{03} \sqrt[3]{w_{2}}\right), \\
I_{1,1}\left(t_{k}, w_{0}, w_{1}, w_{2}\right)=\frac{1}{\left(t_{k}\right)^{3}}\left(\lambda_{11}\left(w_{0}\right)+\lambda_{12} \sqrt[3]{w_{1}}\right), \\
I_{2,1}\left(t_{k}, w_{0}, w_{1}, w_{2}\right)=\frac{1}{\left(t_{k}\right)^{3}}\left(\lambda_{21} \sqrt[3]{w_{0}}+\lambda_{22}\left(w_{1}\right)+\lambda_{23}\left(w_{2}\right)+\operatorname{sgn}\left(w_{2}\right) 5 t_{k}\right) .
\end{gathered}
$$




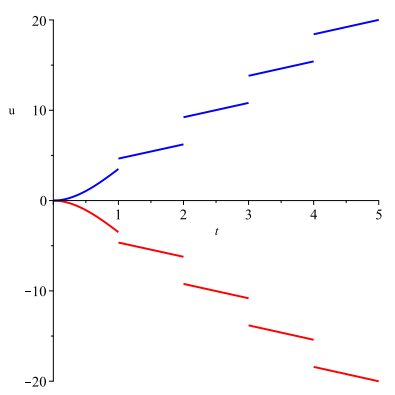

Figure 1. Solution region.

As a numeric example, let us consider $b=-1, A=B=0, t_{k}=k, k \in \mathbb{N}$, and adequate values for the parameters.

In this case, the impulsive conditions are given by

$$
\begin{aligned}
\Delta u(k) & =\frac{1}{k^{3}}\left(0.001^{k} \sqrt[3]{u(k)}+0.001^{k}\left(u^{\prime}(k)\right)+0.001^{k} \sqrt[3]{u^{\prime \prime}(k)}\right), \\
\Delta u^{\prime}(k) & =\frac{1}{k^{3}}\left(0.1^{k}(u(k))+0.1^{k} \sqrt[3]{u^{\prime}(k)}\right), \\
\Delta u^{\prime \prime}(k) & =\frac{1}{k^{3}}\left(-0.1^{k} \sqrt[3]{u(k)}-0.1^{k}\left(u^{\prime}(k)\right)+0.1^{k}\left(u^{\prime \prime}(k)\right)+\operatorname{sgn}\left(u^{\prime \prime}(k)\right) 5 k\right),
\end{aligned}
$$

and the piecewise functions $\alpha, \beta \in X$ defined as

$$
\begin{aligned}
& \alpha(t)= \begin{cases}1.5 t^{3}-5 t^{2}, & \text { if } 0 \leq t \leq 1, \\
-0.1\left(\frac{1}{2 t}+16 t\right)-3 k, & \text { if } t \in] k, k+1], k \geq 1,\end{cases} \\
& \beta(t)= \begin{cases}-1.5 t^{3}+5 t^{2}, & \text { if } t \leq 1, \\
0.1\left(\frac{1}{2 t}+16 t\right)+3 k, & \text { if } t \in] k, k+1], k \geq 1,\end{cases}
\end{aligned}
$$

are, respectively, lower and upper solutions of problem (4.2), (4.3), (4.6), according to Definition 3, satisfying (3.1).

Moreover, the nonlinear part given by (4.5) verifies (3.2), the impulsive functions $I_{i, 1}: \mathbb{R}^{4} \mapsto \mathbb{R}, i=0,1,2$,

$$
\begin{aligned}
& 0,1\left(k, w_{0}, w_{1}, w_{2}\right)=\frac{1}{k^{3}}\left(0.001^{k} \sqrt[3]{w_{0}}+0.001^{k} w_{1}+0.001^{k} \sqrt[3]{w_{2}}\right), \\
& I_{1,1}\left(k, w_{0}, w_{1}, w_{2}\right)=\frac{1}{k^{3}}\left(0.1^{k} w_{0}+0.1^{k} \sqrt[3]{w_{1}}\right), \\
& I_{2,1}\left(k, w_{0}, w_{1}, w_{2}\right)=\frac{1}{k^{3}}\left(-0.1^{k} \sqrt[3]{w_{0}}-0.1^{k} w_{1}+0.1^{k} w_{2}+\operatorname{sgn}\left(w_{2}\right) * 5 k\right),
\end{aligned}
$$

satisfy (3.3), (3.4) and (3.5), and assumption $(A)$ holds for $\xi \geq 24.245$.

Then, by Theorem 2, there exists a solution $u \in X$ of problem (4.2), (4.3), (4.6), in the strip

$$
\alpha(t) \leq u(t) \leq \beta(t), \text { for } t \in(0,+\infty),
$$

that is, in the region illustrated in Figure 1. 
From the localization part of Theorem 2 we can also have some data on the first and second derivatives:

$$
\left.\left.\alpha^{\prime}(t) \leq u^{\prime}(t) \leq \beta^{\prime}(t), \alpha^{\prime \prime}(t) \leq u^{\prime \prime}(t) \leq \beta^{\prime \prime}(t), \text { for } t \in\right] 0,+\infty\right),
$$

that is the growth and concavity variations are in the strips given by Figure 2 .

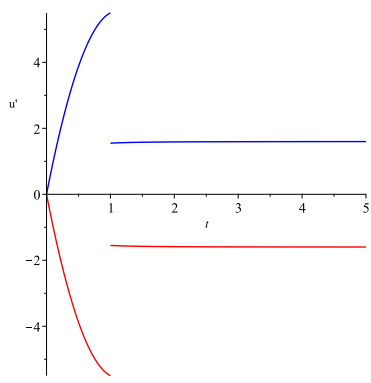

a)

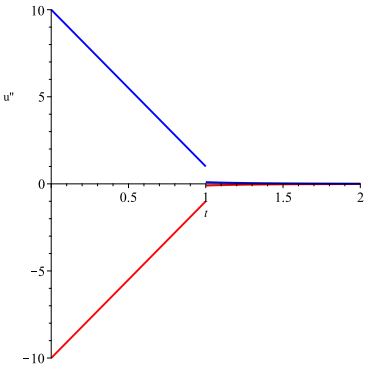

b)

Figure 2. a) derivative region; b) concavity variation.

\section{Conclusions}

Higher-order boundary value problems on unbounded domains are more delicate, as the nonlinearities can be chaotic. From a theoretical point of view, the issue relies on the noncompacity of the associated operator, and to overcome it, some additional tools are required, such as the stability at $\infty$. Moreover, in impulsive problems, with extra jumps, the stability on each impulsive moment must hold. Lower and upper solutions prove to be an adequate method and technique for these kinds of problems, as it gives not only the existence of a solution but also some qualitative data such as monotonicity, variations, concavity,.... In this way, it enlarges the range of applications and it is very useful for nonlinear problems, where it is not possible to have an explicit solution.

\section{References}

[1] R.P. Agarwal and D. O'Regan. Multiple nonnegative solutions for second order impulsive differential equations. Appl. Math. Comput, 155:51-59, 2000. https://doi.org/10.1016/S0096-3003(99)00074-0.

[2] R.P. Agarwal and D. O'Regan. Infinite Interval Problems for Differential, Difference and Integral Equations. Kluwer Academic Publisher, Glasgow, 2001. https://doi.org/10.1007/978-94-010-0718-4.

[3] R.P. Agarwal and D. O'Regan. Non-linear boundary value problems on the semi-infinite interval: an upper and lower solution approach. Mathematika, 49(12):129-140, 2002. https://doi.org/10.1112/S0025579300016120.

[4] C. Bai and C. Li. Unbounded upper and lower solution method for third-order boundary-value problems on the half-line. Electronic Journal of Differential Equations, 2009(119):1-12, 2009. https://doi.org/10.1023/A:1021167932414. 
[5] F. Bernis and L.A. Peletier. Two problems from draining flows involving thirdorder ordinary differential equations. SIAM J. Math. Anal., 27:515-527, 1996. https://doi.org/10.1137/S0036141093260847.

[6] A. Cabada, F. Minhós and A.I. Santos. Solvability for a third order discontinuous fully equation with functional boundary conditions. J. Math. Anal. Appl., 322:735-748, 2006. https://doi.org/10.1016/j.jmaa.2005.09.065.

[7] T. Chiam. Stagnation-point flow towards a stretching plate. J. Phys. Soc. Jpn., 63:2443-2444, 1994. https://doi.org/10.1143/JPSJ.63.2443.

[8] C. Corduneanu. Integral Equations and Stability of Feedback Systems. Academic Press, NewYork, 1973.

[9] J. Graef, L. Kong and F. Minhós. Higher order boundary value problems with $\Phi$ Laplacian and functional boundary conditions. Computers and Mathematics with Applications, 61:236-249, 2011. https://doi.org/10.1016/j.camwa.2010.10.044.

[10] J. Graef, L. Kong, F. Minhós and J. Fialho. On the lower and upper solutions method for higher order functional boundary value problems. Applicable Analysis and Discrete Mathematics, 5(1):133-146, 2011. https://doi.org/10.2298/AADM110221010G.

[11] H. Lian, P. Wang and W. Ge. Unbounded upper and lower solutions method for Sturm-Liouville boundary value problem on infinite intervals. Nonlinear Anal., 70:2627-2633, 2009. https://doi.org/10.1016/j.na.2008.03.049.

[12] Y. Liu. Existence of solutions of boundary value problems for coupled singular differential equations on whole lines with impulses. Mediterr. J. Math., 12:697716, 2015. https://doi.org/10.1007/s00009-014-0422-1.

[13] Y. Liu and D. O'Regan. Multiplicity results using bifurcation techniques for a class of boundary value problems of impulsive differential equations. Commun. Nonlinear Sci. Numer. Simul., 16:1769-1775, 2011. https://doi.org/10.1016/j.cnsns.2010.09.001.

[14] R. Ma, B. Yang and Z. Wang. Positive periodic solutions of first-order delay differential equations with impulses. Appl. Math. Comput., 219:6074-6083, 2013. https://doi.org/10.1016/j.amc.2012.12.020.

[15] T.R. Mahapatra and A.S. Gupta. Heat transfer in stagnation-point flow towards a stretching sheet. Heat Mass Transfer, 38:517-521, 2002. https://doi.org/10.1007/s002310100215.

[16] F. Minhós and R. Carapinha. Half-linear impulsive problems for classical and singular $\varphi$-Laplacian with generalized impulsive conditions. Journal of Fixed Point Theory and Applications, 20(3):117, 2018. https://doi.org/10.1007/s11784-0180598-2.

[17] F. Minhós and H. Carrasco. High order boundary value problems on unbounded domains: Types of solutions, functional problems and applications: Trends in abstract and applied analysis. World Scientific, 5, 2017. https://doi.org/10.1007/s002310100215.

[18] F. Minhós and H. Carrasco. Lidstone-type problems on the whole real line and homoclinic solutions applied to infinite beams. Neural Computing \& Application, 2020. https://doi.org/10.1007/s00521-020-04732-x.

[19] F. Minhós and R. de Sousa. Solvability of coupled systems of generalized Hammerstein-type integral equations in the real line. Mathematics, 8(111), 2020. https://doi.org/10.3390/math8010111. 
[20] R. Nazar, N. Amin, D. Filip and I. Pop. Stagnation point flow of a micropolar fluid towards a stretching sheet. Int. J. Non-Linear Mech., 39:1227-1235, 2004. https://doi.org/10.1016/j.ijnonlinmec.2003.08.007.

[21] J. Nieto and R. López. Boundary value problems for a class of impulsive functional equations. Comput. Math. Appl., 55:2715-2731, 2008. https://doi.org/10.1016/j.camwa.2007.10.019.

[22] J. Paullet and P. Weidmaz. Analysis of stagnation point flow toward a stretching sheet. International Journal of Non-Linear Mechanic., 42(9):1084-1091, 2007. https://doi.org/10.1016/j.ijnonlinmec.2007.06.003.

[23] M. Reza and A.S. Gupta. Steady two-dimensional oblique stagnation-point flow towards a stretching surface. Fluid Dyn. Res., 37:334-340, 2005. https://doi.org/10.1016/j.fluiddyn.2005.07.001.

[24] A.M. Samoilenko and N.A. Perestyuk. Impulsive Differential Equations. World Scientific, Singapore, 1955. https://doi.org/10.1142/2892.

[25] Y. Tian and W. Ge. Variational methods to Sturm-Liouville boundary value problem for impulsive differential equations. Nonlinear Analysis: Theory, Methods \& Applications, 72:277-287, 2010. https://doi.org/10.1016/j.na.2009.06.051.

[26] W.C. Troya. Solutions of third-order differential equations relevant to draining and coating flows. SIAM J. Math. Anal., 24:155-171, 1993. https://doi.org/10.1137/0524010.

[27] E.O. Tuck and L.W. Schwartz. A boundary value problem from draining and coating flows involving a third-order differential equation relevant to draining and coating flows. SIAM Rev., 32:453-469, 1990. https://doi.org/10.1137/1032079.

[28] J. Xiao, J. Nieto and Z. Luo. Multiplicity of solutions for nonlinear second order impulsive differential equations with linear derivative dependence via variational methods. Communications in Nonlinear Science and Numerical Simulation, 17:426-432, 2012. https://doi.org/10.1016/j.cnsns.2011.05.015.

[29] Y. Xu and H. Zhang. Positive solutions of an infinite boundary value problem for nth-order nonlinear impulsive singular integro-differential equations in Banach spaces. Applied Mathematics and Computation, 218:5806-5818, 2012. https://doi.org/10.1016/j.amc.2011.11.100.

[30] B. Yan, D. O'Regan and R.P. Agarwal. Unbounded solutions for singular boundary value problems on the semi-infinite interval: Upper and lower solutions and multiplicity. J.Comput.Appl.Math., 197:365-386, 2006. https://doi.org/10.1016/j.cam.2005.11.010. 